\title{
Research of Equivalent Testing Intensity Between Proving Grounds Based on Rain-Flow Technique and Miner Theorem
}

\author{
Weiqiang Yue ${ }^{1,2}$ and Chuanxue Song ${ }^{1}$ \\ ${ }^{1}$ Jilin University State Key Laboratory of Automobile Simulation and Control, Changchun, China \\ ${ }^{2}$ Institute of Military Transportation, Tianjin, China
}

\begin{abstract}
Use the LMS data acquisition terminal and the PCB acceleration sensor, get the loading spectrum of two typical proving grounds by the same truck, and then make a contrast analysis of the distributing character by the rain-flow counting technique. At the same time extrapolate the two spectrums into the same testing mileage, then make the fatigue damage analysis of them by the LMS TecWare base on the modified Miner rule, contrast the result we can get the equivalent fatigue damage of the two proving grounds. So we get a key method to have evaluations and regulations in road testing.
\end{abstract}

Keywords-loading spectrum; rain-flow counting; minertheorem; equivalent fatigue damage

\section{INTRODUCTION}

In most new vehicle development activity, the road simulation test plays a very important role by its convenience flexible and efficiency. Usually the vehicle's durability test uses the loading spectrum of proving grounds, because its character road can usually examine some specifical parts of the vehicle very pertinence. But an other questions appears, that is we have some different proving grounds which are build science 1980s, such as the Hainan proving ground, the Dingyuan proving ground and so on[1]. Because the difference of the criterions and design, the main character such as intensity, length and layout of different proving grounds often shows most difference, which put forward a bother to the test engineers, that is how to make a evaluation between the different roads or the different proving grounds. So this paper aimed at solving this problem from researching on the test intensity contrast of two roads by different proving grounds, using several technical measure to analyze the distinct loading spectrum of different road by the same truck, and then give a equivalent test intensity base the modified Miner rule, this research provides a foundation of the road simulation test.

\section{A Survey of Two Proving Grounds AND the LOADING SPECTRUM’S ACQUISITION}

\section{A. A Survey of Two Proving Grounds}

Aimed at the examining heavy truck's road reliability, we choose two representative proving grounds as pertinence, because they are so different. One is constructed by its landform, which needn't many manmade character roads, and its most consumers are the remodeled or special used cars. But the other has more manmade roads such as the stone road the washboard road the Belgium road and so on. Comparing with the first proving ground, roads of the second one are more rigour, and we can forecast entirely different test result for the same truck from the two proving grounds. But the most important thing is how to scale them, so you have to keep on reading us.

\section{B. The Loading Spectrum'S Acquisition}

The equipment is the LMS SCM05 which uses the Text. Xpress software, we use them to acquisition acceleration transferred by 6 PCB sensors which are fixed on the end of truck's 6 wheels' axes. The truck is loading at its $80 \%$ full scale, and then we drive it through all the character roads separately in the two proving grounds. And the always by the same standard velocity which is $50 \mathrm{~km} / \mathrm{h}$ in the ecumenical road and $20 \mathrm{~km} / \mathrm{h}$ in the extremity hard road. Then we can get the loading spectrum of the two proving grounds by the same truck, and the Figure I show the acceleration of left front wheel's axle which is analyzed by replacing burr removing singular points and wiping off the trend item.

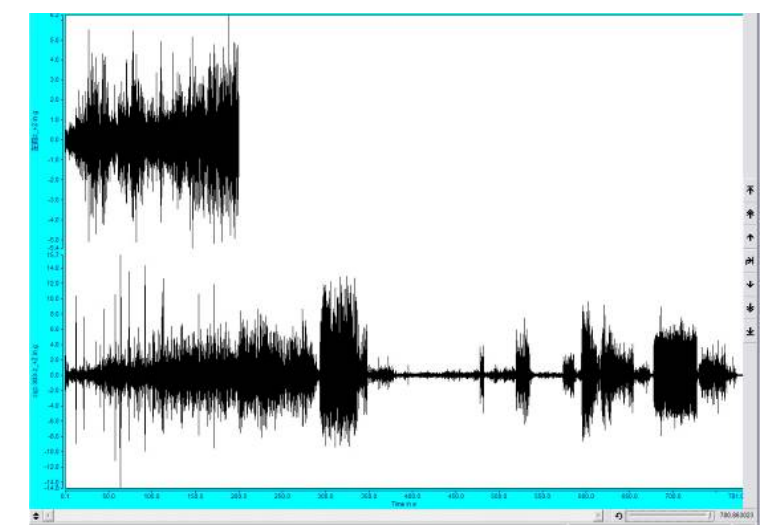

FIGURE I. TWO LOADING SPECTRUM BY THE SAME TRUCK'S LEFT FRONT AXLE IN Z COORDINATE

In the Figure I, the first part is loading spectrum date from proving ground No.1, we call it data A, the second one is from proving ground No.2, we call it data B. we can get some apparent difference of two dates. The average acceleration of Date A is about $2 \mathrm{~g}$, and the extremism is $5 \mathrm{~g}$, its total time is 200s, we can't find intense diversification in it only because there are not so many characteristic roads. On the other hand, the average acceleration of Date $B$ is about $5 \mathrm{~g}$, and the extremum is $14 \mathrm{~g}$, its total time is $780 \mathrm{~s}$, we easily find there are 
many characteristic roads in the proving ground from the distinct boundary of the date.

\section{THE CONTRAST ANALYSIS OF THE LOADING SPECTRUM BY RAIN-FLOW COUNTING}

In the durability test, the most factors of fatigue damage are contributed by the stress amplitude and the cycle number[2]. Base on this theory, the time-domain signal's counting method analysis appears. The counting method is a process translates the load stress into some full cycles and half cycles, and then we can get the load cycles frequency. Normally the counting method includes one parameter counting and two parameters counting, and the rein-flow counting method is most efficient.

\section{A. The Rain-Flow Counting Method}

This rain-flow counting is also called tower top method, which is put forward by two English engineers Matsuiski and Endo. They consider that plastic deformation is the necessary condition to cause fatigue damage, and plastic character shows the delay regression line of stress and strain. The full cycle is the mark of the fatigue damage, presume small delay regression line don't affect the large degree cycle, after taking out the small cycles in order, then we can get the stress and strain counting result under the effect of amplitude of variation load[3-4].
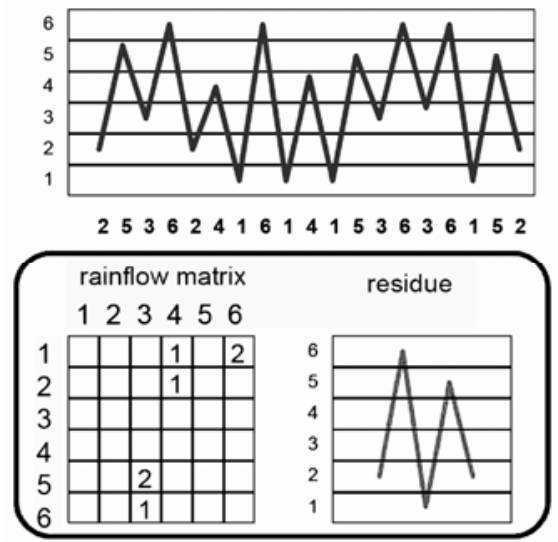

FIGURE II. THE PRINCIPLE OF RAIN-FLOW COUNTING METHOD

So we can get the principle of the rain-flow counting, a rain-flow cycle corresponding to a stress and strain delay, and any time-domain signal can be translated into a rain-flow matrix and a residue, just shown as Figure II. The method can count all the full stress and strain cycles whereas they are large and small, and it is very precise and convent. The result of the method is easily to be statistic by computer counting which is widely used in the fatigue damage research.

\section{B. The Rain-Flow Counting of Two Different Loading Spectrum Dates}

Base on the rain-flow counting theory, use the rain-flow counting analyzer of LMS TecWare to process the two different loading spectrum dates, then we can get their $\mathrm{Z}$ coordinate rain-flow matrix, just shown as Figure III and Figure IV.

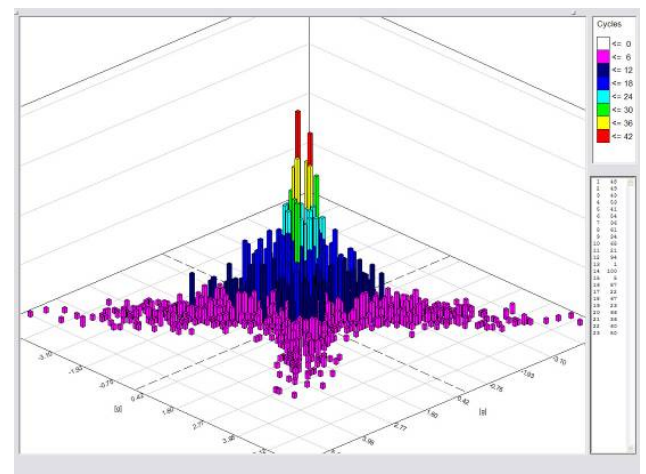

FIGURE III. THE RAIN-FLOW MATRIX OF DATE A

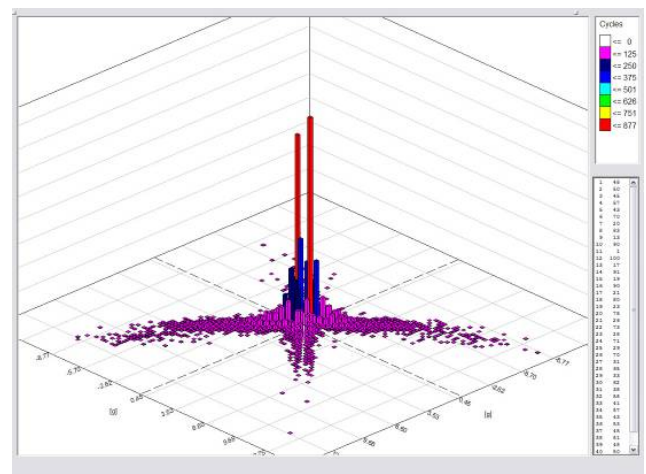

FIGURE IV. THE RAIN-FLOW MATRIX OF DATE B

From the Figure III, we can discover that the date is translated into 4623 rain-flow cycles, which divide the loading amplitude of $-5.45 \mathrm{~g} \sim 6.30 \mathrm{~g}$ into 100 grids, and the highest frequency is 42. From the figure IV, we can discover that the date is translated into 10448 rain-flow cycles, which divide the loading, amplitude of-14.93g 15.83g into 100 grids, and the highest frequency is 877 . We can get some conclusions from a rough analysis.

Firstly, the amplitude range of two dates are very distinct, the amplitude range of date $\mathrm{B}$ is almost triple of the date $\mathrm{A}$, and there are more rain-flow cycles in date B only because it is more longer.

Secondly, the high frequency region are smaller loading cycles, especially for the data $\mathrm{B}$, its small loading cycles are 20 times than date $\mathrm{A}$, the reason is there is a smooth ramp road in proving ground No.2, which produce fewer fatigue damage to the truck, so we can compress this part of the date to reduce the test course by rain-flow compiling.

\section{The Equivalent Fatigue damage Analysis of Two LOADING SPECTRUM}

From the analyze result above, we can easily find out the distinct of two proving ground. So we want to give out a method to quantify the equivalent fatigue damage of them, which play a fatal role in the test formulating. The equivalent fatigue damage analysis is based on the damage theory, which express the damage in numerical values, then relate them in some equivalent. 


\section{A. The Fatigue Damage Cumulation Theory}

Most invalidation of the auto parts are caused by fatigue, which is the conception of the fatigue damage. Consider when the truck running, the continuous loading from the ground make damage to some of its parts. In the mechanics of materials, when a metallic part endures a stress over its yield value, there will be some damage to it which can not be recovered. With more and more damage cumulated to the critical value, the part will be broke, this is the fatigue damage cumulation theory [5-7], and the process is called fatigue life. There are many methods to research the fatigue life, generally speaking, there two types, one is nominal stress method, and the other is local stress and strain method [8].

Miner liner fatigue damage cumulation theory is a simpler and convenient method, which is more popular used in the truck and its parts, the basical theory is shown below. Consider the extreme value of the vibration energy is $W$, the total fatigue cycle is $N$, and the value of No. $n_{1}$ is $W_{1}$, so there is a proportional relation between the loading cycles and absorbed energy, that is $\frac{W_{1}}{W}=\frac{n_{1}}{N}$.

If the stress sequence is $S_{1}, S_{2}, S_{3} \cdots S_{n}$, and the corresponding fatigue life sequences is $N_{1}, N_{2}, N_{3} \cdots N_{\text {n }}$, the loading cycle sequence is $\mathrm{n}_{1}, \mathrm{n}_{2}, \mathrm{n}_{3} \cdots \mathrm{n}_{\mathrm{n}}$, according to the theory above, we can get the fatigue damage cumulation .

$$
D=\frac{n_{1}}{N_{1}}+\frac{n_{2}}{N_{2}}+\frac{n_{3}}{N_{2}}+\cdots+\frac{n_{1}}{N_{1}}=\stackrel{\circ}{\mathrm{a}} \frac{\mathrm{n}_{\mathrm{i}}}{N_{\mathrm{i}}}
$$

When $D=1$, the part is broken.

Base on the equivalent fatigue damage theory, use the analyzer of LMS TecWare to process the two different loading spectrum dates, then we can get their $\mathrm{Z}$ coordinate fatigue damage. Because the mileage of two proving grounds is not the same, so we should extrapolate them to the same extent to establish the equivalence. Then as shown if Figure V, indicate the equivalent fatigue damage of two proving ground by the same test mileage.

\section{B. The Equivalent Fatigue Damage Analysis}

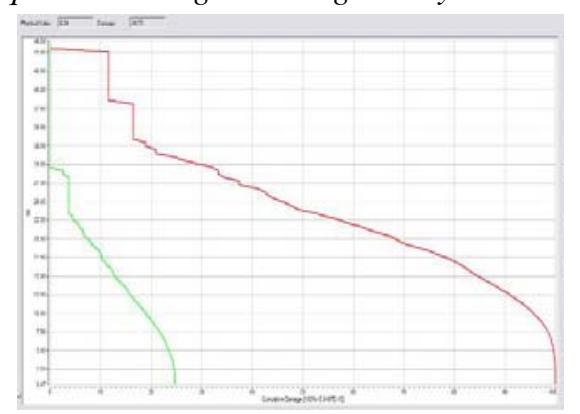

FIGURE V. THE CONTRAST ANALYSIS OF TWO LOADING SPECTRUM BY EQUIVALENT FATIGUE DAMAGE ANALYSIS
Table I shows the equivalent fatigue damage of the loading dates by two proving grounds in 6 axle's $\mathrm{Z}$ coordinate.

TABLE I. 6 AXLE'S Z COORDINATES EQUIVALENT FATIGUE DAMAGE

\begin{tabular}{|c|c|c|c|}
\hline Item & $\begin{array}{c}\text { Date1 } \\
\text { (E-15) }\end{array}$ & $\begin{array}{c}\text { Date2 } \\
\text { (E-15) }\end{array}$ & $\begin{array}{c}\text { equivalent fatigue } \\
\text { damage }\end{array}$ \\
\hline Front left & 0.96067 & 3.2687 & 0.29 \\
\hline Front right & 1.2462 & 4.0497 & 0.31 \\
\hline Middle left & 1.3499 & 4.3791 & 0.31 \\
\hline Middle right & 0.75637 & 2.3299 & 0.32 \\
\hline Rear left & 0.72074 & 2.1518 & 0.33 \\
\hline Rear right & 0.87442 & 2.4340 & 0.36 \\
\hline \multicolumn{3}{|c|}{ Average value } \\
\hline
\end{tabular}

From the Table I, we can get the equivalent fatigue damage of two loading spectrum, which also shows the test intensity of two proving ground to the same truck. We can get the conclusion that the damage of proving ground No.1 is 32\% of proving ground No.2, which means to the same truck under the same test mileage, it endure the 3times fatigue damage.

\section{CONCLUSIONS}

This paper aimed at the test intensity of different roads in two proving grounds, driving the same truck to get the loading spectrum dates. Use the time-domain contrasting and rein-flow counting to analyze the difference. Then based on the Miner liner fatigue damage cumulation theory, we get the equivalent fatigue damage of two proving ground, which give the important precondition to formulate the testing program and evaluate the testing result, also put forward a feasible solution to this kind of problem.

\section{REFERENCES}

[1] Li Peng. Research on enhancement test coefficient for automobile proving ground [D]. Changchun; Jilin university, 2007:5-7.

[2] Gao Zhen-tong, Xiong Jun-jiang. Fatigue reliability [M]. Beijing, Beijing university of aeronautics and astronautics press, 2000:23-26.

[3] Khosrovanch A K and Dowling N E. Fatigue Loading History Reconstruction Based on the Rain-flow Technique [J]. International Journal of Fatigue, 1990(2) : 21-24.

[4] S.D.Downing and D.F.Socie, Simple rain-flow counting algorithms[J]. INT.J.FATIGUE, 1982(1) : 31-38.

[5] Zhao Shao-bian. Study on the accuracy of fatigue life predictions by the generally used damage cumulation theory [J]. Journal of mechanical strength, 2000.9(Vol22.3):31 33.

[6] Xu Hao. Fatigue intensity [M]. Beijing higher education press, 1998 : 24 26.

[7] Zhao Hong-zhi. A discussion about the application of Miner theorem [J]. Journal of machine design, 2007.8(24):10 12.

[8] Yu Chang-qing. Research of equivalent coefficient of automobile reliability test on road simulation system of MTS [D]. Changchun; Jilin university, 2006.10: 43 44. 